\title{
The first pancreatic neuroendocrine tumor in Li-Fraumeni syndrome: a case report
}

\author{
John G. Aversa', Francine Blumental De Abreu², Sho Yano ${ }^{3}$, Liqiang Xi ${ }^{2}$, Donald W. Hadley ${ }^{4}$, Irini Manoli ${ }^{3}$,
} Mark Raffeld ${ }^{2}$, Samira M. Sadowski ${ }^{1}$ and Naris Nilubol ${ }^{1 *}$

\begin{abstract}
Background: Li-Fraumeni syndrome is a cancer predisposition syndrome caused by germline TP53 tumor suppressor gene mutations, with no previous association with pancreatic neuroendocrine tumors (PNETs). Here we present the first case of PNET associated with Li-Fraumeni syndrome.

Case presentation: This is a 43-year-old female who underwent laparoscopic distal pancreatectomy at age 39 for a well-differentiated grade 2 cystic PNET. When the patient was 41 years old, her seven-year-old daughter was found to have an astrocytoma and a germline TP53 mutation. While undergoing surveillance with ${ }^{68} \mathrm{Gallium}$-DOTATATE positron emission tomography/computed tomography for her PNET, the patient was found to have a large choroid plexus papilloma in her right temporal lobe. She underwent genetic counseling and testing that identified a germline pathogenic variant in TP53, leading to the diagnosis of Li-Fraumeni syndrome. Her PNET had a hemizygous pathogenic TP53 mutation with loss of the wild-type alternate allele, consistent with loss of heterozygosity and the two-hit hypothesis. She was enrolled in a Li-Fraumeni syndrome protocol and continues surveillance screening with our service.
\end{abstract}

Conclusions: This is the first PNET reported in association with Li-Fraumeni syndrome. Pancreatic cancer risk is elevated in this syndrome, and our case highlights the need for vigilance in screening for pancreatic neoplasms in these patients.

Keywords: Pancreatic neuroendocrine tumor, Li-Fraumeni syndrome, TP53, Hereditary pancreatic neoplasm

\section{Background}

Pancreatic neuroendocrine tumors (PNETs) are a rare histologic subtype of pancreatic neoplasm, with an incidence of approximately $0.32 / 100,000$ people in the United States [1, 2]. Although most PNETs occur sporadically, there are four inherited syndromes associated with them: neurofibromatosis type 1 (NF1 gene; OMIM \#162200), von Hippel-Lindau disease (VHL gene; OMIM \#193300), multiple endocrine neoplasia type 1 (MEN1 gene; OMIM \#131100), and tuberous sclerosis complex (TSC1 and TSC2 genes; OMIM \#191100 and \#191092,

\footnotetext{
* Correspondence: niluboln@mail.nih.gov

'Surgical Oncology Program, Center for Cancer Research, National Cancer Institute, National Institutes of Health, 10 Center Drive, Building 10, Room 4-5952, Bethesda, MD 20892, USA

Full list of author information is available at the end of the article
}

respectively). Li-Fraumeni syndrome (LFS) is a rare hereditary cancer predisposition syndrome caused by a heterozygous TP53 germline mutation [3]. LFS has established associations with breast, brain, adrenal, hematological, and colorectal cancers, along with bone and soft tissue sarcomas, but to date no association with PNET has been reported [4].

Although somatic TP53 mutations have been identified in various grades of PNETs as well as gastric, small bowel, colorectal, and appendiceal neuroendocrine tumors (NETs), there are no reported cases of NETs associated with a germline TP53 mutation $[2,5,6]$. TP53 mutations are commonly found in poorly differentiated neuroendocrine carcinomas [2]. TP53 is a tumor suppressor gene located on locus 17p13.1 that codes for p53, the most commonly inactivated protein in human 
cancer [7]. Here we report a patient who initially presented with cystic PNET and later was found to have LFS. We performed next-generation sequencing of the PNET, demonstrating a "second-hit" on TP53, which is the first reported manifestation of PNET in association with LFS [8].

\section{Case presentation}

The patient is a 43-year-old female who underwent laparoscopic distal pancreatectomy in 2015 (age 39) after a long-standing history of a large $(12.7 \times 11 \times 10 \mathrm{~cm})$ septated pancreatic cyst with a nodular, thickened wall (Fig. 1). She initially presented with mild discomfort in her left upper abdomen in 2013 (age 37). The physical exam was unremarkable. Imaging studies revealed a large, heterogeneous pancreatic tail cyst, along with a 6 $\mathrm{cm}$ heterogeneous left kidney lesion most consistent with angiomyolipoma and a $2.5 \mathrm{~cm}$ right liver lesion consistent with hemangioma. Surgical resection of the pancreatic cyst was recommended, but the patient instead elected to undergo transgastric endoscopic fineneedle aspiration at an external facility in 2014. The cyst recurred (found on follow-up imaging), and she then underwent laparoscopic distal pancreatectomy in 2015.

Pathologic examination was consistent with a World Health Organization (WHO) well-differentiated grade 2 PNET (the Ki-67 proliferation index was $3.5 \%$, and the mitotic rate was $<1 / 50$ high-powered fields). The tumor cells stained positive for chromogranin and synaptophysin. The patient continued surveillance visits and imaging, with no evidence of recurrence.

In 2017, her seven-year-old daughter was diagnosed with a WHO grade 3 isocitrate dehydrogenase wild-type intracranial astrocytoma with a TP53 somatic mutation identified in the tumor. A germline pathogenic mutation

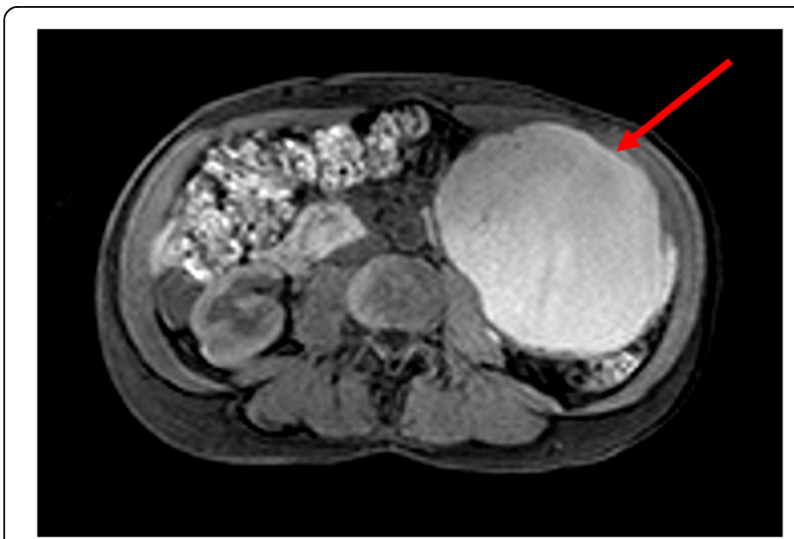

Fig. 1 Cross-sectional imaging of cystic PNET. MRI demonstrating a nearly $13 \mathrm{~cm}$ cystic pancreatic lesion (identified with the red arrow) that was resected along with a portion of the distal pancreas in 2015. Histopathology showed a World Health Organization grade 2 well-differentiated neuroendocrine tumor in TP53 was also identified, but exact variant information was not available from the testing laboratory. Parental testing was not performed at the time. Shortly after diagnosis, she died from tumor progression.

In 2018 (age 42), the patient underwent a ${ }^{68}$ GalliumDOTATATE positron emission tomography/computed tomography (PET/CT) scan for PNET surveillance, which revealed a large right temporal lobe mass that was further characterized on brain magnetic resonance imaging (MRI) to represent a large $(5.3 \times 3.3 \times 4.3 \mathrm{~cm})$ enhancing intraventricular mass causing mass effect and midline shift (Fig. 2).

In light of this finding and her daughter's history, the patient was evaluated by neurosurgical and genetic counseling teams. She underwent craniotomy and resection for the intracranial lesion. Pathologic examination showed that the mass was a WHO grade 1 choroid plexus papilloma, that stained positive for synaptophysin, glial fibrillary acidic protein, cell adhesion molecule 5, p53, and cytokeratin 7. Table 1 shows a timeline of major events in the patient's care.

The patient's paternal ancestry is Russian, and her maternal ancestors are non-Ashkenazi Russian Jewish. Her maternal grandmother's fraternal twin sister died at age 65 of a rare gastrointestinal (GI) cancer, and her paternal grandmother had uterine cancer in her 50s. The detailed history of the patient's other family members, particularly on her father's side, is unknown.

The patient underwent genetic testing in a Clinical Laboratory Improvement Amendments-certified molecular genetics laboratory (Invitae, San Francisco, CA) and was found to carry a pathogenic germline TP53 mutation, namely a cytosine-to-thymine transition at codon 1009, leading to an arginine-to-cysteine substitution (c.1009C > T, p.R337C). Initial surveillance exams and studies were performed according to MD Anderson Cancer Center's LFS Education and Early Detection Adult Screening Guidelines [9].

Our patient's care plan includes a yearly whole-body MRI, a yearly breast thermogram (the patient refused a mammogram) and breast MRI, a yearly brain MRI, abdominal and pelvic ultrasounds every 6 months, esophagogastroduodenoscopy and colonoscopy every two to 5 years, and yearly labs. Additionally, the patient will undergo a clinical breast exam every 6 months and a yearly skin exam. She will continue with a yearly abdominal MRI for PNET and angiomyolipoma surveillance.

Surveillance detected no conclusive evidence of additional neoplasms, but it revealed several indeterminate osseous lesions that are currently being monitored.

Paraffin-embedded PNET tissue from 2015 was assayed for a profile of common somatic mutations (Oncomine Comprehensive Assay v3, Thermo Fisher Scientific, Carlsbad, CA) and found to have a 


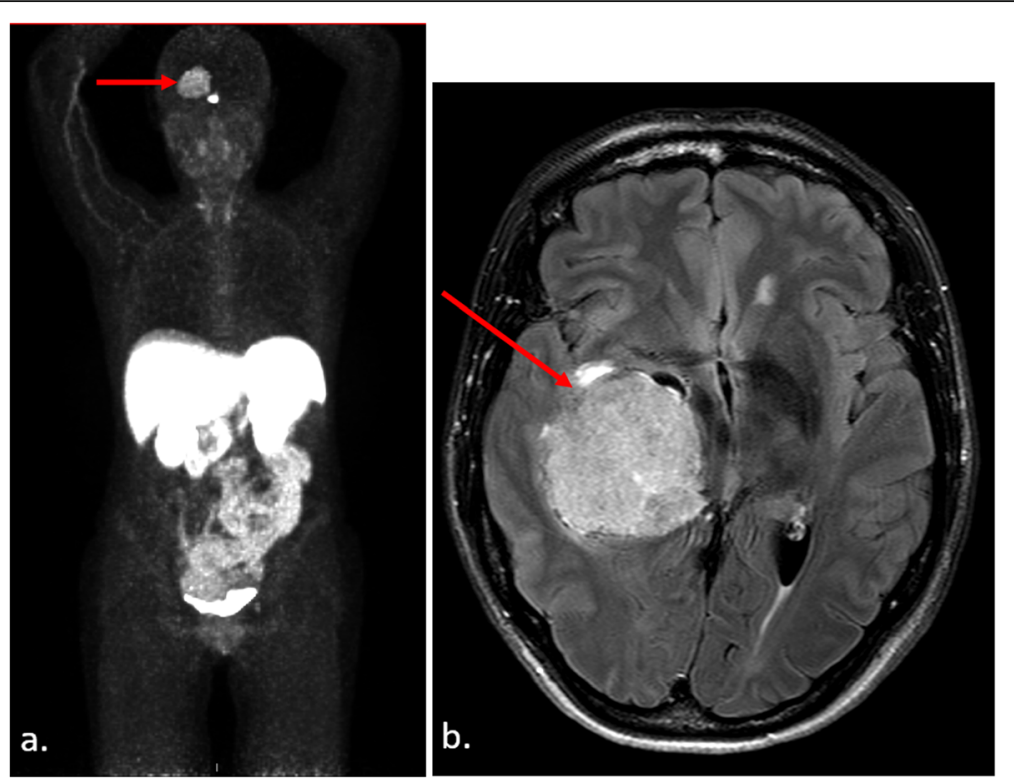

Fig. $2{ }^{68}$ Gallium-DOTATATE PET/CT study revealing a right-sided, avid intracranial mass. a. Planar whole-body maximum-intensity projection, with red arrow identifying the intracranial mass. b. Fluid-attenuated inversion recovery (FLAIR) post-contrast brain MRI demonstrating a $5.3 \times 3.3 \times 4.3$ $\mathrm{cm}$ enhancing intraventricular mass (identified with red arrow) in the temporal horn of the right ventricle, which was associated with nearcomplete compression of the right lateral ventricle and $6 \mathrm{~mm}$ midline shift at the level of the foramen of Monro

hemizygous pathogenic TP53 mutation at codon 1009 (NM_000546.5: c.1009C > T, p.R337C). Variant allele frequency was $93 \%$, consistent with loss of the wild-type alternate allele (loss of heterozygosity) as a "second hit" in the tumor suppressor gene.

\section{Discussion and conclusions}

Here we report the first PNET in association with LFS. Next-generation sequencing reveals a hemizygous pathogenic TP53 mutation (p.R337C) consistent with a loss of heterozygosity at the locus. This represents a novel finding that expands the clinical spectrum of LFS. Although TP53 somatic mutations are frequently found in neuroendocrine carcinoma, they do not commonly appear in well-differentiated NET with prolonged disease-free survival, such as in this patient $[2,5,6]$.

Prior studies demonstrate somatic TP53 mutations in all grades of NET from various primary sites, with high-

Table 1 Important dates in the case

\begin{tabular}{ll}
\hline Date & Event \\
\hline $8 / 2013$ & Began to develop symptoms of abdominal discomfort \\
$3 / 2014$ & MRI showing large cystic mass in pancreatic tail \\
$8 / 2014$ & Endoscopic aspiration of pancreatic tail cyst \\
$3 / 2015$ & Laparoscopic distal pancreatectomy/splenectomy \\
$8 / 2017$ & Patient's daughter diagnosed with astrocytoma \\
$10 / 2018$ & Underwent craniotomy and resection for right temporal \\
$2 / 2019$ & lobe choroid plexus papilloma detected on PET/CT \\
\hline
\end{tabular}

grade tumors more commonly harboring the mutation in both intra-abdominal and extra-abdominal NETs. Overall, more than half of poorly differentiated GI NECs have somatic TP53 mutations, while $0-11 \%$ of moderately and well-differentiated GI NETs harbor the mutation $[2,5,10,11]$. Similarly, for pulmonary NETs, more than $60 \%$ of small cell lung cancer and large cell neuroendocrine carcinoma have TP53 mutations, and 0-11\% of well-differentiated grade 1 and 2 pulmonary NETs have been shown to have TP53 mutations [12, 13].

None of these prior studies that reported somatic mutations in NETs included germline mutation sequencing for their study cohorts. Our report demonstrates not only the next-generation sequencing of our PNET verifying a hemizygous TP53 mutation and loss of the wildtype allele, but also shows our patient's TP53 germline mutation with other clinical manifestations consistent with LFS.

Although the prevalence and the risk of developing PNET in patients with LFS is unknown, this patient population has a known predisposition for pancreatic adenocarcinoma at a rate of approximately seven times that of the general population [14]. Our patient had no additional pathogenic mutations detected by the Oncomine Comprehensive Assay, and we postulate that a "second-hit" somatic TP53 mutation in the setting of an existing germline TP53 mutation drove the development of her PNET. However, it is possible that there are additional pathogenic somatic mutations that were not included in this assay. 
In the setting of a new PNET diagnosis, current guidelines advocate for either recommendation (in the case of $V H L, T S C$, and NF1) or consideration (in the case of $M E N 1)$ of genetic testing when a patient has a personal and/or family history compatible with the known associated syndromes [15]. Similar advice is reasonable here, as the majority of PNETs are sporadic and this is the first described case associated with LFS.

Our patient's angiomyolipoma in the context of her LFS diagnosis warrants further consideration. She follows up with the urologic surgery service for surveillance, and although she and the service have discussed a biopsy to determine the presence of malignant features, she has opted for imaging surveillance at this time. Although angiomyolipomas are normally benign lesions, there have been reports of malignant angiomyolipoma in LFS patients, which emphasizes the importance of surveillance and, potentially, the need for intervention [16].

The current clinical criteria (Chompret criteria) to identify suspected LFS patients who are candidates for genetic testing centers around presentation according to family history, multiple primary tumors, rare tumors, or early-onset breast cancer [17]. Familial criteria for testing include typical LFS-associated tumors (osteosarcoma, soft tissue sarcoma, central nervous system tumors, premenopausal breast cancer, and adrenocortical carcinoma) diagnosed in the proband at an age younger than 46 years, as well as at least one first- or second-degree relative who presents with an LFS-related tumor before age 56 or who has multiple tumors [17]. The multiple primary tumor criteria require that the proband presents with multiple primary tumors (except breast cancer), with two of the tumors belonging to the LFS tumor spectrum, the first of which must appear before age 46 [17]. The rare tumor criteria include any patient with adrenocortical carcinoma, choroid plexus tumor, or the embryonal anaplastic subtype of rhabdomyosarcoma [17]. Patients who present with breast cancer before 31 years of age are recommended to undergo TP53 germline mutation testing as well [17]. Although most PNETs present in patients 50 years old or above, it is not uncommon for patients to present at a younger age $[18,19]$. Only in conjunction with additional findings or a family history suggestive of LFS would we recommend testing for a TP53 germline mutation in new diagnosis of PNET.

Our findings in this case would not change the surveillance program for pancreatic neoplasm screening in LFS (yearly whole-body MRI under many LFS screening protocols). We recommend a dedicated abdominal MRI in LFS patients to increase sensitivity in the detection of pancreatic neoplasms. This is also commonly recommended in LFS screening guidelines, including the MD Anderson high-risk pancreatic cancer screening guidelines [20]. In this particular situation, where a patient has manifested PNET in association with LFS, we recommend a dedicated abdominal MRI every 6 months for 2 years after resection, and yearly thereafter if there remains no evidence of disease. Interpreting the relevance of our patient's development of PNET to her specific TP53 mutation (p.R337C) is difficult, as genotypephenotype relationships in LFS are not fully characterized [17, 21, 22]. Prior analyses have categorized TP53 pathogenic variants by genetic location and by predicted structural and/or functional properties of mutant proteins. Missense mutations affecting residues belonging to loops 2 and 3 of the p53 protein were found to be associated with the development of brain tumors [14, 23]. Missense mutations affecting residues outside of the loop-sheet-helix motif that binds the major groove of target DNA in p53 were found to be associated with the development of adrenocortical carcinomas [14, 23].

Attempts have been made to classify TP53 mutation pathogenic variant with LFS phenotype. Ideally, this would provide insight for more targeted screening for specific malignancies in these carriers. Although genotypic variation has not been shown to correlate with the development of particular tumors, dominant-negative missense TP53 mutations leave their carriers susceptible to cancer at earlier ages and at higher levels of clinical severity than those with non-missense mutations [17]. Despite the limitations of our finding in the broad scope of LFS, it is important to consider when developing more targeted screening protocols for specific LFS genotypes.

Germline TP53 mutations most commonly occur within the DNA-binding domain, leading to the production of a dysfunctional p53 protein [24]. Our patient's particular TP53 mutation is relatively uncommon and has initially been implicated in LFS and Li-Fraumeni-like syndromes [25]. A cytosine-to-thymine transition at nucleic acid 1009 leads to a missense arginine-to-cysteine transition at amino acid 337 (p.R337C) within exon 10. This results in p53 alteration along its basic C-terminus, leading to a reduction in overall functioning p53 levels; tetramerization fraction; and, therefore, its DNA-binding ability and transactivation [26, 27]. The p.R337C germline mutation has been seen in cohorts that tend to develop brain tumors, osteosarcoma, rhabdomyosarcoma, breast cancer, and childhood adrenocortical carcinoma [25, 27-31].

A prior analysis established three age-based categories along a "cancer spectrum" of LFS [32]. Classification included tumors belonging to the "childhood phase" (adrenocortical carcinoma, choroid plexus carcinoma, rhabdomyosarcoma, and medulloblastoma), "early adulthood phase" (breast cancer, osteosarcoma, leukemia, astrocytoma, glioblastoma, colorectal cancer, and other sarcomas [malignant fibrous histiocytoma, liposarcoma, leiomyosarcoma]), and "late adulthood phase" (pancreatic and prostate cancer) [32]. Genotypic variation in LFS has been best 
correlated with a phenotype in Brazilian families that harbor the more common TP53 germline mutation that features a transition from guanine to adenine and a subsequent arginine-to-histidine transition (c.1010G > A, p.R337H), rendering carriers particularly susceptible to the development of adrenocortical carcinoma [31].

This patient's case represents the first reported PNET in association with a germline TP53 mutation, highlighting the importance of screening for pancreatic neoplasms in the setting of LFS. The p.R337C mutation is an uncommon pathogenic variant of TP53 in LFS, and much remains to be described about its associated cancers. To aid patient surveillance programs, further studies are warranted to elucidate a genotype-phenotype correlation and the other molecular mechanisms associated with LFS phenotypes. This case report was prepared following the CARE guidelines and methodology [33].

\section{Abbreviations}

c.1009C > T: Mutation at codon 1009 cytosine to thymine; c.1010G >

A: Mutation at codon 1010 guanine to adenine; CT: Computed tomography; FLAIR: Fluid-attenuated inversion recovery; Gl: Gastrointestinal; LFS: LiFraumeni syndrome; MEN1: Multiple endocrine neoplasia type 1; MRI: Magnetic resonance imaging; NET: Neuroendocrine tumor; NF1: Neurofibromatosis type 1; p.R337C: Mutation at amino acid 337 arginine to cysteine; p.R337H: Mutation at amino acid 337 arginine to histidine; PET: Positron emission tomography; PNET: Pancreatic neuroendocrine tumor; TSC: Tuberous sclerosis complex; VHL: Von Hippel-Lindau; WHO: World Health Organization

\section{Acknowledgements}

This research was supported by the Intramural Research Program of the NIH, National Cancer Institute.

\section{Authors' contributions}

JA and NN assembled, analyzed, and interpreted the patient data and contributed significantly to the writing of the manuscript. SS was a major contributor in writing the manuscript. IM, SY, and DH were major contributors in coordinating patient care and writing the manuscript. $F B, L X$, and MR interpreted the patient data regarding the Oncomine Comprehensive Assay and were major contributors in writing the manuscript. All authors read and approved the final manuscript.

\section{Funding}

The National Cancer Institute at the National Institutes of Health provided all funding for the design of the study and the collection, analysis, and interpretation of the data.

\section{Availability of data and materials}

Supporting data can be found in the Clinical Research Information System, which is the electronic medical record at the National Institutes of Health. These data are not available publicly due to patient privacy restrictions but are available from the corresponding author upon reasonable request.

\section{Ethics approval and consent to participate}

The patient provided written informed consent, and the study was approved by the National Cancer Institute's Institutional Review Board.

The full document explaining the study, with the patient's signature for consent, is available from the corresponding author upon reasonable request.

\section{Consent for publication}

The patient provided written informed consent for publication of her clinical details and images and the clinical details of her deceased family members. A copy of the consent form is available for review by the editor of this journal.

\section{Competing interests}

The authors declare that they have no competing interests.

\section{Author details}

${ }^{1}$ Surgical Oncology Program, Center for Cancer Research, National Cancer Institute, National Institutes of Health, 10 Center Drive, Building 10, Room 4-5952, Bethesda, MD 20892, USA. 'Laboratory of Pathology, Center for Cancer Research, National Cancer Institute, National Institutes of Health, Bethesda, MD 20892, USA. ${ }^{3}$ Medical Genomics and Metabolic Genetics Branch, National Human Genome Research Institute, National Institutes of Health, Bethesda, MD 20892, USA. ${ }^{4}$ Human Development Section, Medical Genetics Branch, National Human Genome Research Institute, National Institutes of Health, Bethesda, MD 20892, USA.

Received: 13 September 2019 Accepted: 6 March 2020

Published online: 30 March 2020

\section{References}

1. Yao JC, Hassan M, Phan A, Dagohoy C, Leary C, Mares JE, Abdalla EK, Fleming JB, Vauthey JN, Rashid A, et al. One hundred years after "carcinoid": epidemiology of and prognostic factors for neuroendocrine tumors in 35,825 cases in the United States. J Clin Oncol. 2008;26(18):3063-72.

2. Vijayvergia N, Boland PM, Handorf E, Gustafson KS, Gong Y, Cooper HS, Sheriff F, Astsaturov I, Cohen SJ, Engstrom PF. Molecular profiling of neuroendocrine malignancies to identify prognostic and therapeutic markers: a fox Chase Cancer Center pilot study. Br J Cancer. 2016;115:564.

3. Malkin D, Li FP, Strong LC, Fraumeni JF Jr, Nelson CE, Kim DH, Kassel J, Gryka MA, Bischoff FZ, Tainsky MA, et al. Germ line p53 mutations in a familial syndrome of breast cancer, sarcomas, and other neoplasms. Science. 1990;250(4985):1233-8.

4. Guha T, Malkin D. Inherited TP53 Mutations and the Li-Fraumeni Syndrome. Cold Spring Harb Perspect Med. 2017;7(4).

5. Park HY, Kwon MJ, Kang HS, Kim YJ, Kim NY, Kim MJ, Min KW, Choi KC, Nam ES, Cho SJ, et al. Targeted next-generation sequencing of well-differentiated rectal, gastric, and appendiceal neuroendocrine tumors to identify potential targets. Hum Pathol. 2019.

6. Jiao Y, Shi C, Edil BH, de Wilde RF, Klimstra DS, Maitra A, Schulick RD, Tang LH, Wolfgang CL, Choti MA, et al. DAXX/ATRX, MEN1, and mTOR pathway genes are frequently altered in pancreatic neuroendocrine tumors. Science. 2011;331(6021):1199-203.

7. Pflaum J, Schlosser S, Muller M. p53 family and cellular stress responses in Cancer. Front Oncol. 2014;4:285.

8. Knudson AG Jr. Mutation and cancer: statistical study of retinoblastoma. Proc Natl Acad Sci U S A. 1971;68(4):820-3.

9. Amini B, Bevers T, Bojadzieva J, Coyne R, Day S, Jackson T, Strong L, Thomas P, Williams A, Willis B, Yang S. MD Anderson Li-Fraumeni syndrome screening - adult. 2017. https://www.mdanderson.org/content/dam/ mdanderson/documents/for-physicians/algorithms/screening/screening-lifraumeni-web-algorithm.pdf. Accessed 30 Aug 2019.

10. Park C, Ha SY, Kim ST, Kim HC, Heo JS, Park YS, Lauwers G, Lee J, Kim KM. Identification of the BRAF V600E mutation in gastroenteropancreatic neuroendocrine tumors. Oncotarget. 2016;7(4):4024-35.

11. Yachida S, Vakiani E, White CM, Zhong Y, Saunders T, Morgan R, de Wilde RF, Maitra A, Hicks J, Demarzo AM, et al. Small cell and large cell neuroendocrine carcinomas of the pancreas are genetically similar and distinct from well-differentiated pancreatic neuroendocrine tumors. Am J Surg Pathol. 2012;36(2):173-84.

12. Simbolo M, Mafficini A, Sikora KO, Fassan M, Barbi S, Corbo V, Mastracci L, Rusev B, Grillo F, Vicentini C, et al. Lung neuroendocrine tumours: deep sequencing of the four World Health Organization histotypes reveals chromatin-remodelling genes as major players and a prognostic role for TERT, RB1, MEN1 and KMT2D. J Pathol. 2017;241(4):488-500.

13. Walter RF, Vollbrecht C, Christoph D, Werner R, Schmeller J, Flom E, Trakada G, Rapti A, Adamidis V, Hohenforst-Schmidt W, et al. Massive parallel sequencing and digital gene expression analysis reveals potential mechanisms to overcome therapy resistance in pulmonary neuroendocrine tumors. J Cancer. 2016;7(15):2165-72.

14. Ruijs MW, Verhoef S, Rookus MA, Pruntel R, van der Hout AH, Hogervorst FB, Kluijt I, Sijmons RH, Aalfs CM, Wagner A, et al. TP53 germline mutation testing in 180 families suspected of Li-Fraumeni syndrome: mutation 
detection rate and relative frequency of cancers in different familial phenotypes. J Med Genet. 2010;47(6):421-8.

15. Kunz PL, Reidy-Lagunes D, Anthony LB, Bertino EM, Brendtro K, Chan JA, Chen $\mathrm{H}$, Jensen RT, Kim MK, Klimstra DS, et al. Consensus guidelines for the management and treatment of neuroendocrine tumors. Pancreas. 2013; 42(4):557-77.

16. Li J, Zhu M, Wang YL. Malignant epithelioid angiomyolipoma of the kidney with pulmonary metastases and p53 gene mutation. World J Surg Oncol. 2012;10:213.

17. Bougeard G, Renaux-Petel M, Flaman JM, Charbonnier C, Fermey P, Belotti M, Gauthier-Villars M, Stoppa-Lyonnet D, Consolino E, Brugieres L, et al. Revisiting Li-Fraumeni syndrome from TP53 mutation carriers. J Clin Oncol. 2015;33(21):2345-52.

18. Halfdanarson TR, Rabe KG, Rubin J, Petersen GM. Pancreatic neuroendocrine tumors (PNETs): incidence, prognosis and recent trend toward improved survival. Ann Oncol. 2008;19(10):1727-33.

19. Ben Q, Zhong J, Fei J, Chen H, Yv L, Tan J, Yuan Y. Risk factors for sporadic pancreatic neuroendocrine tumors: a case-control study. Sci Rep. 2016;6:36073.

20. Bannon S, Bevers T, Bhutani M, Brown P, McGuire M, Fleckenstein ON, Hawk E, Katz M, McAllister F, Pai A. MD Anderson pancreatic cancer screening. 2017. https://www.mdanderson.org/content/dam/mdanderson/documents/ for-physicians/algorithms/screening/screening-pancreatic-web-algorithm. pdf. Accessed 28 Aug 2019.

21. FP LI, FRAUMENI JF JR. Soft-tissue sarcomas, breast Cancer, and other neoplasms: a familial syndrome? Ann Intern Med. 1969;71(4):747-52.

22. Tinat J, Bougeard G, Baert-Desurmont S, Vasseur S, Martin C, Bouvignies E, Caron O, Bressac-de Paillerets B, Berthet P, Dugast C, et al. Version of the Chompret criteria for Li Fraumeni syndrome. J Clin Oncol. 2009;27(26):e1089 author reply e10.

23. Olivier M, Goldgar DE, Sodha N, Ohgaki H, Kleihues P, Hainaut P, Eeles RA. Li-Fraumeni and related syndromes: correlation between tumor type, family structure, and TP53 genotype. Cancer Res. 2003;63(20):6643-50.

24. Pinto EM, Ribeiro RC, Figueiredo BC, Zambetti GP. TP53-associated pediatric malignancies. Genes Cancer. 2011;2(4):485-90.

25. Luca JW, Strong LC, Hansen MF. A germline missense mutation R337C in exon 10 of the human p53 gene. Hum Mutat. 1998;(Suppl 1):S58-61.

26. Kamada R, Nomura T, Anderson CW, Sakaguchi K. Cancer-associated p53 tetramerization domain mutants: quantitative analysis reveals a low threshold for tumor suppressor inactivation. J Biol Chem. 2011;286(1):252-8.

27. Jordan JJ, Inga A, Conway K, Edmiston S, Carey LA, Wu L, Resnick MA. Altered-function p53 missense mutations identified in breast cancers can have subtle effects on transactivation. Mol Cancer Res. 2010;8(5):701-16.

28. Barnes DM, Hanby AM, Gillett CE, Mohammed S, Hodgson S, Bobrow LG, Leigh IM, Purkis T, MacGeoch C, Spurr NK, et al. Abnormal expression of wild type p53 protein in normal cells of a cancer family patient. Lancet. 1992;340(8814):259-63.

29. Lomax ME, Barnes DM, Gilchrist R, Picksley SM, Varley JM, Camplejohn RS. Two functional assays employed to detect an unusual mutation in the oligomerisation domain of p53 in a Li-Fraumeni like family. Oncogene. 1997:14:1869.

30. Andrade RC, Dos Santos AC, de Aguirre Neto JC, Nevado J, Lapunzina P, Vargas FR. TP53 and CDKN1A mutation analysis in families with Li-Fraumeni and Li-Fraumeni like syndromes. Familial Cancer. 2017;16(2):243-8.

31. Achatz Ml, Zambetti GP. The Inherited p53 Mutation in the Brazilian Population. Cold Spring Harb Perspect Med. 2016;6(12).

32. Amadou A, Waddington Achatz MI, Hainaut P. Revisiting tumor patterns and penetrance in germline TP53 mutation carriers: temporal phases of LiFraumeni syndrome. Curr Opin Oncol. 2018;30(1):23-9.

33. Gagnier JJ, Kienle G, Altman DG, Moher D, Sox H, Riley D. The CARE guidelines: consensus-based clinical case reporting guideline development. Glob Adv Health Med. 2013;2(5):38-43.

\section{Publisher's Note}

Springer Nature remains neutral with regard to jurisdictional claims in published maps and institutional affiliations.

\section{Ready to submit your research? Choose BMC and benefit from:}

- fast, convenient online submission

- thorough peer review by experienced researchers in your field

- rapid publication on acceptance

- support for research data, including large and complex data types

- gold Open Access which fosters wider collaboration and increased citations

- maximum visibility for your research: over $100 \mathrm{M}$ website views per year

At BMC, research is always in progress.

Learn more biomedcentral.com/submissions 